analysis of families of non-syndromic cleft lip probands from three human populations, which the authors claim supports major gene determination of liability and not the multifactorial threshold model; and lastly a paper which includes helpful information for counselling in van der Woude syndrome families.

The third section, perhaps the most interesting, deals with teratogenesis. The effects of hypoxia and ethanol on facial development in experimental animals are reported and there are several papers on the effects of vitamin A derivatives.

The last section, as last sections often are, is heterogeneous, with papers including necropsy data on Japanese newborns and fetuses, trisomic mice, rat medial edge epithelium (beautifully illustrated), an extraordinary human fetus, and those Burmese cats! This is a book to dip into rather than to read through. It would earn its place in a university library, where it would be consulted by as varied a group of people as the papers it contains. It has been well edited and the tables, diagrams, and illustrations are of a high standard.

Dian DonnaI

\section{Veterinary Genetics}

By F W Nicholas. (Pp 580; £29.50.) Oxford: Oxford Science Publications. 1987.

Let me start by confessing my ignorance of veterinary genetics. I would not presume to judge how well Nicholas's book stands comparison with other books on the subject, but it is very interesting for an ignorant clinical geneticist to peer over the fence and see what is going on next door.

At first sight, the neighbours live very much like us. Chapters on basic genetics, recombinant DNA, biochemical genetics, chromosome aberrations, and immunogenetics offer a good modern treatment of familiar material. You could mine them for recondite facts suitable for one-upmanship. ("It is of course well known that fox squirrels all have red bones and teeth because of their extremely low uroporphyrinogen III cosynthetase activity-would you like to comment on why they don't have clinical porphyria?".) Some of these recondite facts remind us that the genetic arrangements we see in humans may be evolutionary quirks rather than biological imperatives. On the whole, though, these chapters emphasise the similarity between veterinary and human clinical genetics.

Later sections go into breed structure, crossing $\overline{0}$ strategies, and methods of selection, matters of obvious importance to animal breeders but not to $\frac{\bar{m}}{\frac{1}{5}}$ us. The pharmacogenetics section is also rather $\mathbb{\varnothing}$ different from a human version, being chiefly concerned with how best to poison mice. I had been particularly interested to look for two topics, animal. models and multifactorial inheritance. On animal $\overrightarrow{\vec{\omega}}$ models there was relatively little, because veterinary $\underset{\sigma}{\omega}$ genetics is mainly concerned with farm animals and the most interesting models are usually in mire. Multifactorial inheritance was thoroughly interesting. of Increasingly, as I go yet again through the motions or of teaching multifactorial threshold theory $10 \mathrm{~W}$ students, I find myself wondering whether this is not one of the great genetic myths. The mathematics is $\frac{3}{3}$ very impressive, but what can it actually do? For $\overrightarrow{\vec{z}}$ humans, the answer is probably "very little", and maybe we should stop burdening students with a formalism which is guaranteed to produce a minimum $\vec{\varphi}$ of useful insight for a maximum of sweat and tears. Animal geneticists, to judge by this book, are not 0 troubled by such doubts, because they need mathematical tools for guiding breeding experiments. To quote Nicholas, "by thinking in terms of a multifactorial model we can forget about detecting $\frac{2}{\square}$ carriers or removing a gene from the population, $\underset{\vec{P}}{\overrightarrow{2}}$ and concentrate instead on more fruitful tasks ... 응 multifactorial inheritance is known and understood, and appropriate breeding plans for the reduction in frequency of a particular defect or disease can easily be drawn up and put into practice on the basis of that understanding".

That quotation summarises for me a crucia! $\frac{0}{3}$ difference between human and veterinary genetics. For us, the unit is the individual patient, for vets it is the breed. They do not share the clinician's preoccupation with the isolated abnormal person. Chromosomal translocations, for example, are seen $\frac{7}{0}$ mainly as a cause of reduced fertility, not of abnormal offspring. Population genetics is corres- $N$ pondingly more important, and they need to be $\mathrm{N}$ better at maths than we do. Overall what we see is the same knowledge being applied to a rather $\omega$ different purpose, and it is the difference in purpose rather than any difference in material which marks $\stackrel{\circ}{\subset}$ the distinction between veterinary and human clinical genetics.

ANDREW P READ $\frac{0}{\stackrel{0}{\circ}}$ 